\title{
Standard Free Thickness Determination of Thin TEM Samples via Backscatter Electron Image Correlation
}

\author{
R. Salzer, A. Graff, M. Simon, F. Altmann
}

Fraunhofer Institute for Mechanics of Materials, Walter-Hülse-Strasse 1, 06120 Halle Germany

Due to the ongoing progress in nanotechnology, high resolution TEM investigations become more and more important in structural and chemical material analyses. For such investigations the thickness of the TEM sample should be as thin as possible. The thickness of TEM samples prepared by focused ion beam (FIB) is about 50 to $100 \mathrm{~nm}$. For light elements like silicon this might be sufficient for most of the analytical work. Due to the Z-dependence of the electron scattering it is also necessary to achieve even smaller thicknesses to perform TEM analyses at materials with high mass densities or high atomic numbers like $\mathrm{Au}$ or $\mathrm{Pt}$. The difficulties to prepare plane-parallel lamellae with thicknesses of 10 to $50 \mathrm{~nm}$ by FIB are known. Common methods especially the FIB preparation do not have the ability to control the thickness precisely during the preparation process. Therefore, it is difficult or nearly impossible to stop the FIB sputtering process at the right target thickness.

The aim of the presented work was to use the contrast formation of backscattered electrons (BSE) at thin samples by SEM during FIB preparation to determine the thickness of the observed TEM sample. The images produced by backscattered electrons have the advantage that they are not influenced by any FIB generated electrons. Thus, BSE can be used to monitor the thickness changes whilst FIB milling of TEM samples. The contrast in a backscattered electron image of a thin sample depends among other things on the maximal depth from which electrons could be backscattered. If the thickness of a sample is smaller than this depth a part of the primary electrons, which would be backscattered in bulk samples, will traverse the sample and could not be detected as BSE's. So the amount of BSE's will decrease (see thin edge of the wedge in Fig. 1). This thickness dependend contrast change is also a function of the material of the sample and the energy of the incident electrons. To describe this BSE contrast formation we derive a global normalized backscatter electron transmission function (GNTF). This function depends on primary electron energy, thickness and material parameters (element and density) of the lamella. It is based on a modified regularized incomplete Gamma-function adapted to Monte-Carlo simulations of electron trajectories in solids (CASINO) [1] (Fig. 1 b). The GNTF is used to calculate for each pixel of a BSE-image of a thin sample a thickness value and display all of them in a color-coded thickness map. For this the transmission function is scaled with the maximum (bulk sample area) and the minimum (background without sample) grey values (Fig. 1 a).

To verify the calculated normalized BSE transmission gradients, wedges of different materials were prepared by FIB milling. The thickness determined by analyzing the grey values was compared to values measured at cross sections at certain markers (Fig. 1,2).

\section{References}

[1] D. Drouin et al. Scanning Vol. 29 Issue 3 (2007) 92-101

[2] This work was kindly supported by the Carl Zeiss NTS GmbH 

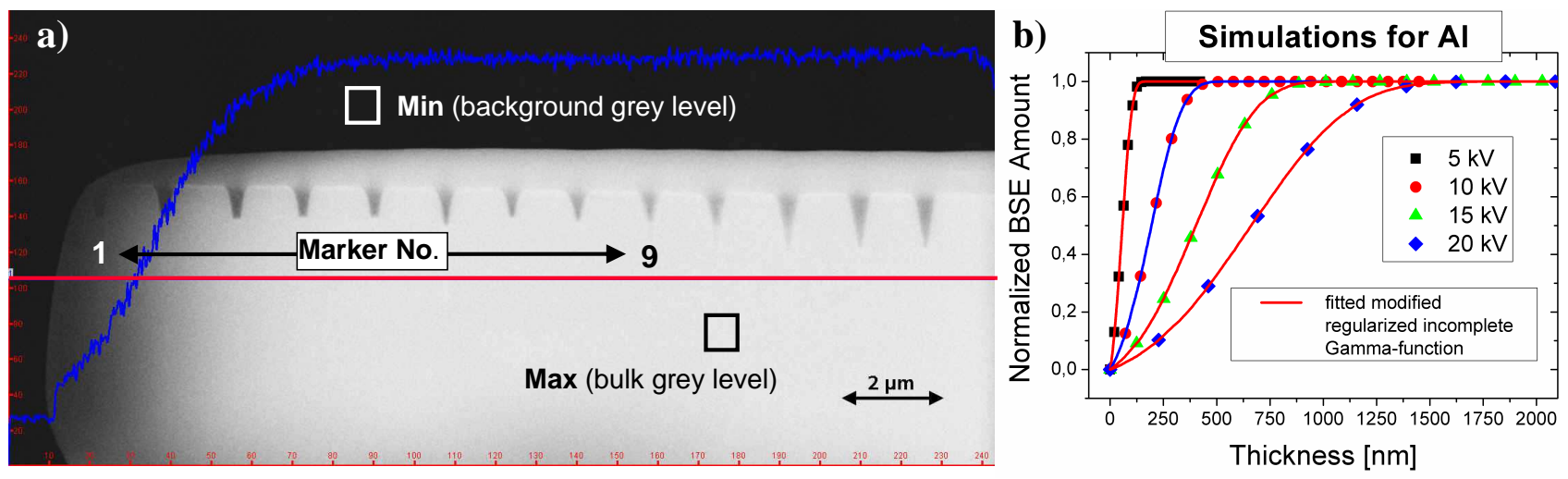

Fig. 1. a) BSE image of an Aluminum wedge with markers at the top observed at $10 \mathrm{kV}$ accelerating voltage. The blue line indicates the grey level at the red horizontal line. The white rectangle shows an area of the minimum and the black one of the maximum grey level. b) The graph represents the simulated normalized amount of BSE's for different acceleration voltages of Aluminum. The blue line shows the simulation for $10 \mathrm{kV}$ as used for imaging in a).
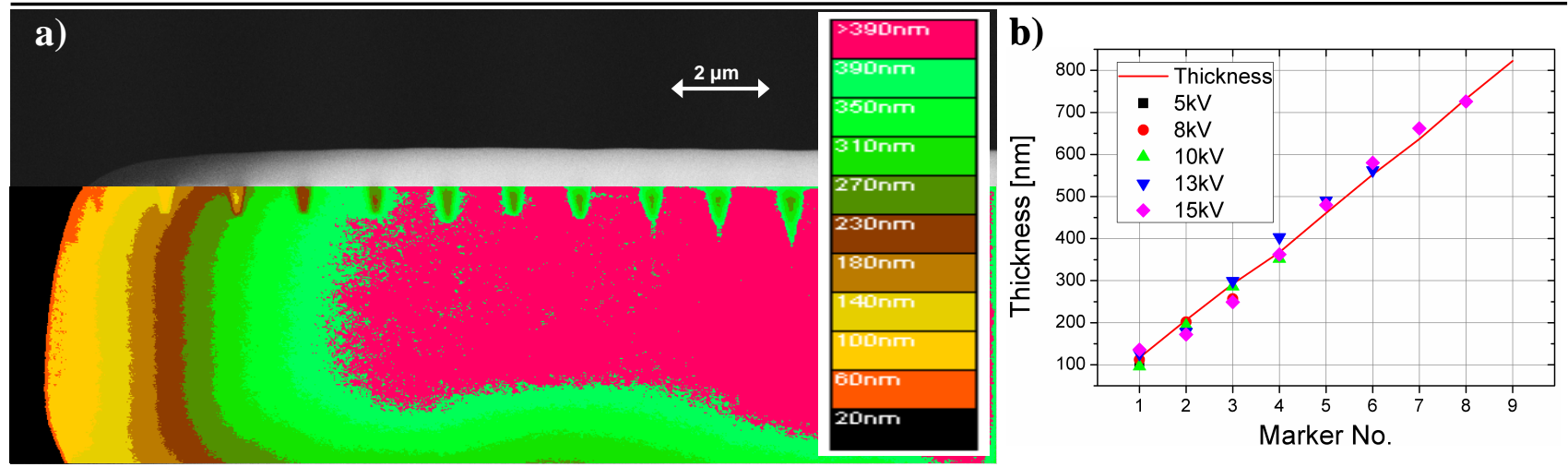

Fig. 2. a) BSE-image of Fig.1 a) overlaid with the color-coded thickness map calculated using the GNTF which is derived from simulations. b) Measured thicknesses at each marker (red line) compared with the thicknesses calculated by grey level analyses at the position of the associated markers (see Fig. 1 Marker No.).

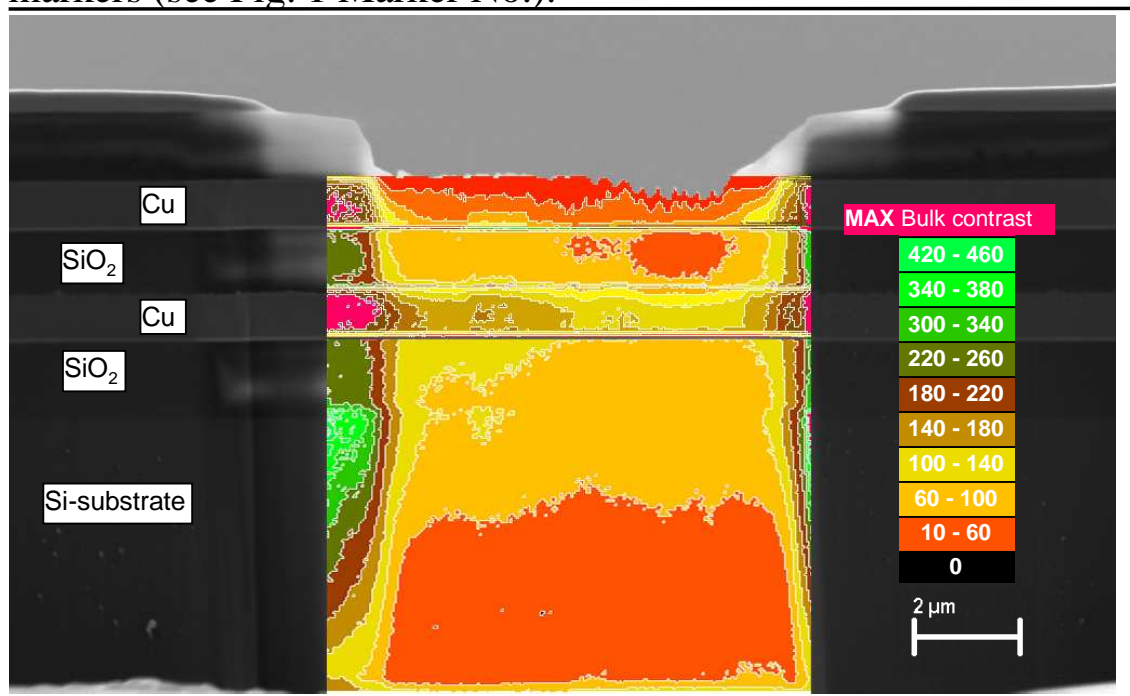

Fig. 3. Example of a secondary electron image of an IC-structure overlaid with the calculated thickness map of the different materials from corresponding BSE-images. 